\title{
Counting models of set theory
}

\author{
by
}

Ali Enayat (Washington, DC)

\begin{abstract}
Let $T$ denote a completion of ZF. We are interested in the number $\mu(T)$ of isomorphism types of countable well-founded models of $T$. Given any countable order type $\tau$, we are also interested in the number $\mu(T, \tau)$ of isomorphism types of countable models of $T$ whose ordinals have order type $\tau$. We prove:

(1) Suppose ZFC has an uncountable well-founded model and $\kappa \in \omega \cup\left\{\aleph_{0}, \aleph_{1}, 2^{\aleph_{0}}\right\}$. There is some completion $T$ of $Z F$ such that $\mu(T)=\kappa$.

(2) If $\alpha<\omega_{1}$ and $\mu(T, \alpha)>\aleph_{0}$, then $\mu(T, \alpha)=2^{\aleph_{0}}$.

(3) If $\alpha<\omega_{1}$ and $T \vdash \mathbf{V} \neq \mathbf{O D}$, then $\mu(T, \alpha) \in\left\{0,2^{\aleph_{0}}\right\}$.

(4) If $\tau$ is not well-ordered then $\mu(T, \tau) \in\left\{0,2^{\aleph_{0}}\right\}$.

(5) If ZFC + "there is a measurable cardinal" has a well-founded model of height $\alpha<\omega_{1}$, then $\mu(T, \alpha)=2^{\aleph_{0}}$ for some complete extension $T$ of $Z F+\mathbf{V}=\mathbf{O D}$.
\end{abstract}

1. Introduction. Countable models of Zermelo-Fraenkel set theory ZF made their public debut in Thoralf Skolem's paper [Sk] in 1923, in which he presented his historic proposal to base axiomatic set theory on first order logic, and in the same breath, unveiled the possibility of an enigmatic miniature universe - a countable model of set theory - obeying the same first order laws as Cantor's galaxy of sets. The deep ramifications of Skolem's "paradox" of set-theoretic ambivalence, however, had to await the revolution in set theory brought about by Paul Cohen's invention of the method of forcing $[C]$ in 1963 and its subsequent elaboration by a generation of set theorists who unearthed a plethora of diverse models of set theory. There is by now a vast set-theoretic literature on models of set theory, often marked by forcing arguments establishing the relative consistency of various assertions, or by explorations of definability and fine structure issues pertaining to inner models, particularly those of large cardinals. The present paper, however, is a modest contribution to the model-theoretic tradition of studying models of set theory, a tradition following in the footsteps of Skolem's pioneering work on "unintended" models of set theory and arithmetic, whose mod-

2000 Mathematics Subject Classification: Primary 03C62, 03C15; Secondary 03H99. 
ern exemplars include Keisler and Morley's [KM], Friedman's [Fr-1], and Ressayre's [Re].

In this paper we study the number of isomorphism types of countable models $\mathfrak{M}$ of arbitrary completions $T$ of ZF when certain second order conditions, such as being well-founded, are imposed on $\mathfrak{M}$. Of course, Gödel's second incompleteness theorem dictates that no model of ZF can be produced using the axioms of ZF alone. However, as soon as we assume the consistency of ZF we can use the Gödel-Rosser incompleteness theorem, asserting the incompleteness of sufficiently expressive recursively enumerable theories, to build a binary tree of sentences of height $\omega$, no two branches of which lie in the same consistent extension of ZF. Hence there are $2^{\aleph_{0}}$ distinct complete extensions of ZF, implying - via the completeness theorem - the existence of continuum many nonisomorphic countable models of ZF. One can even do better: the number of isomorphism types of countable models of each consistent extension $T$ of ZF is also $2^{\aleph_{0}}$ since by a routine compactness argument, any subset of $\omega$ can be included in the standard system (see Definition 2.1) of some countable model $\mathfrak{M}$ of $T$. To conclude:

Proposition 1.1. If $Z F$ is consistent then

(a) ZF has $2^{\aleph_{0}}$ completions;

(b) each consistent completion of ZF has $2^{\aleph_{0}}$ nonisomorphic countable models.

It is therefore natural to inquire about the number of isomorphism types of countable models $\mathfrak{M}$ of $T$ when certain second order constraints are imposed on $\mathfrak{M}$. In particular, one can ask about the number of isomorphism types of:

(1) countable well-founded models of $T$,

(2) countable models of $T$ whose ordinals are isomorphic to a prescribed linear order $\tau$,

(3) countable models of $T$ whose ordinal standard parts are equal to a prescribed ordinal (see Definition 2.1 for ordinal standard part).

We calculate the solution to (1) in Theorem 3.2. A number of results towards the calculation of the solution of (2) when $\tau$ is well-ordered are presented. Here we mention a sample result: If $T$ is a completion of $\mathrm{ZF}+$ $\mathbf{V} \neq \mathbf{O D}$ and $\alpha$ is a countable ordinal, then as soon as there is at least one well-founded model of $T$ of height $\alpha$ then there must be $2^{\aleph_{0}}$ of them (Theorem 3.5). Moreover, assuming the existence of an uncountable wellfounded model of ZFC, there is an unbounded subset $S$ of $\omega_{1}^{\mathrm{L}}$ such that for each $\alpha \in S, L_{\alpha} \vDash \mathrm{ZF}$, and every completion of ZF $+\mathbf{V}=\mathbf{O D}$ has at most one model of height $\alpha$ (Corollary 3.6.2(a) and Theorem 3.7). Corollary 4.3 
settles all the cases in (2) where $\tau$ is not well-ordered, and Corollary 4.2 gives a complete solution of (3).

The paper should be accessible to both set theorists and model theorists. Section 2 is devoted to preliminary definitions and results, all of which (except for Theorem 2.6) come from the literature, or are folklore. We discuss the number of isomorphism types of countable well-founded models in Section 3 , and the number of isomorphism types of countable non-well-founded models in Section 4. Finally in Section 5 we mention further results and list some open questions, the most prominent of which asks whether there exists a complete extension of ZF which has exactly $\lambda$ models of a given ordinal height when $\lambda$ is any cardinal with $2 \leq \lambda \leq \aleph_{0}$.

The initial results obtained in this project go back to my days in graduate school in the mid-1980's during which I had the opportunity to profit from conversations concerning the questions dealt with here with my former advisor Kenneth Kunen, and with H. Jerome Keisler. I am indebted to Kunen for Theorem 3.9, Hugh Woodin for part (b) of Theorem 3.8, Jan Mycielski for numerous helpful suggestions, and to the anonymous referee for a meticulous and insightful report. Finally, I wish to express my gratitude to Roman Kossak and James Schmerl for various helpful discussions regarding models of Peano arithmetic, and for tireless mathematical camaraderie.

2. Preliminaries. We begin with two lists of definitions that will be used throughout the paper. The first list (Definition 2.1) deals with standard notions in the model theory of set theory, and the second one (Definition 2.2) presents the central concerns of this paper.

Definition 2.1. Suppose $\mathfrak{M}=(M, E)$ is a model of ZF, where $E=\in^{\mathfrak{M}}$.

- $\operatorname{Ord}(\mathfrak{M})$ denotes the linearly ordered (by $E$ ) set of "ordinals" of $\mathfrak{M}$. We shall assume without loss of generality that $\omega$ is an initial segment of $\operatorname{Ord}(\mathfrak{M})$.

- A model is standard if it is isomorphic to some $(M, \in)$, where $M$ is a transitive set. When $M$ is transitive we shall use $M$ instead of $(M, \in)$ or $\mathfrak{M}$. (By Mostowski's collapsing lemma, each well-founded model is isomorphic to a unique standard model.) $\mathfrak{M}$ is said to be $\omega$-standard if $\omega^{\mathfrak{M}}$ is well-ordered under $E$ (equivalently: if $\mathfrak{M}$ has no nonstandard integers).

- The standard system of $\mathfrak{M}$, denoted by $\operatorname{SSy}(\mathfrak{M})$, is the collection of all subsets of the form $a_{E} \cap \omega$, where $a \in M$ and $a_{E}=\{m \in M: m E a\}$. (Standard systems play a key role in the structure theory of non- $\omega$-standard models.)

- The ordinal standard part of $\mathfrak{M}$, denoted by osp $(\mathfrak{M})$, is the largest ordinal which can be isomorphically embedded as an initial segment of $\operatorname{Ord}(\mathfrak{M})$. (It is known that ordinal standard parts of models of the Kripke-Platek set 
theory KP are admissible ordinals [Ba, Lemma 8.4]. Moreover, Friedman [Fr-1, Sec. 2] has shown that under reasonable conditions any countable admissible ordinal can be the ordinal standard part of some model of ZF; see also [Ke, p. 60, Corollary C], [Ba, Theorem 7.5].)

- $\varrho$ is the usual ordinal-valued rank function on sets and $V_{\alpha}$ is the $\alpha$ th level of the von Neumann cumulative hierarchy, so $V_{\alpha}=\{x: \varrho(x)<\alpha\}$.

- $\mathbf{V}=\mathbf{O D}$ refers to the sentence in the language of set theory, which expresses - for models of ZF - the statement "every set is first order definable from ordinal parameters". (Models of ZF satisfying $\mathbf{V}=\mathbf{O D}$ are exactly those having a definable global choice function. Furthermore, if $\mathfrak{M} \vDash \mathrm{ZF}$ then the submodel $\mathbf{H O D}^{\mathfrak{M}}$ consisting of hereditarily ordinal definable elements of $\mathfrak{M}$ satisfies ZFC; see [Ku, Ch. 4] for more detail.)

Definition 2.2. Suppose $T$ is a completion of $\mathrm{ZF}, \alpha$ is a countable ordinal, and $\tau$ is a countable linear order.

- $\mathcal{M}(T, \tau)$ is the class of countable models $\mathfrak{M}$ of $T$ such that $\operatorname{Ord}(\mathfrak{M}) \cong \tau$, and $\mu(T, \tau)$ is the number of isomorphism types of models in $\mathcal{M}(T, \tau)$.

- When $\tau$ is $\alpha$ we shall say " $\mathfrak{M}$ has height $\alpha$ " instead of " $\mathfrak{M} \in \mathcal{M}(T, \alpha)$ ".

- $\mathcal{M}(T)$ is the class of countable well-founded models of $T$, and $\mu(T)$ is the number of isomorphism types of models in $\mathcal{M}(T)$. Of course by Mostowski's collapsing lemma $\mu(T)$ is the number of countable transitive models of $T$.

We now record some basic results. Recall that a partial order $\mathbb{P}$ is said to be weakly homogeneous if for any two conditions $p$ and $q$ in $\mathbb{P}$, there is an automorphism $f$ of $\mathbb{P}$ such that $f(p)$ and $q$ are compatible, and $\mathbb{P}$ is said to be perfect if for each $p \in \mathbb{P}$, there are $q, r \in \mathbb{P}$ such that $q$ and $r$ are incompatible and both extend $p$.

Theorem 2.3 (Lévy [J, Theorem 10.19(a)]). If $\mathfrak{M}$ is a model of $Z F$, and $\mathbb{P}$ is a weakly homogeneous partial order in the sense of $\mathfrak{M}$, and $G_{1}$ and $G_{2}$ are $\mathbb{P}$-generic filters over $\mathfrak{M}$, then the generic extensions $\mathfrak{M}\left[G_{1}\right]$ and $\mathfrak{M}\left[G_{2}\right]$ are elementarily equivalent. Moreover, $\mathfrak{M}\left[G_{1}\right] \vDash \mathbf{V} \neq \mathbf{O D}$.

Theorem 2.4 (Folklore). Suppose $M$ is a countable standard model of $Z F$ and $\mathbb{P}$ is a perfect partial order in $M$. There exists a family $\mathcal{G}$ of power $2^{\aleph_{0}}$ of $\mathbb{P}$-generic filters over $M$ such that $M[G] \cap M[\bar{G}]=M$ for distinct $G$ and $\bar{G}$ in $\mathcal{G}$.

Proof. One can construct a binary tree $\left\{p_{s}: s \in 2^{<\omega}\right\}$ of height $\omega$ of conditions whose branches give rise to a family of filters $\left\{G_{f}: f \in 2^{\omega}\right\}=\mathcal{G}$, with $G_{f}=$ the filter generated by $\left\{p_{s}: s \subseteq f\right\}$, such that for any sequence of distinct $f_{1}, \ldots, f_{n}$ in $2^{\omega}$ the product filter $G_{f_{1}} \times \ldots \times G_{f_{n}}$ is $\mathbb{P}^{n}$-generic over $M$. This was done for Cohen forcing by Friedman in [Fr-2, Lemma 8] 
but the construction works for any perfect partial order $\mathbb{P}$. The observation that generic extensions corresponding to mutually generic filters intersect in the ground model is due to Solovay [So, Lemma 2.5].

Theorem 2.5. Suppose $\mathbb{P}$ is a perfect partial order in a countable transitive model $M$ of $Z F$. There exist $2^{\aleph_{0}}$ pairwise nonisomorphic $\mathbb{P}$-generic extensions of $M$.

Proof. This is a direct consequence of Theorems 2.3 and 2.4.

Although many results concerning forcing over models of set theory do not make an essential use of the well-foundedness of the ground model, the proof of Theorem 2.5 above does. Since nonstandard models play a key role in this paper, we elaborate:

TheOREM 2.6. Suppose $\mathfrak{M}$ is a countable recursively saturated model of $Z F$ and $\mathbb{P}$ is a weakly homogeneous partial order in $\mathfrak{M}$. If $G_{1}$ and $G_{2}$ are $\mathbb{P}$-generic filters over $\mathfrak{M}$ then $\mathfrak{M}\left[G_{1}\right] \cong \mathfrak{M}\left[G_{2}\right]$.

Proof. Suppose $G$ is a $\mathbb{P}$-generic filter over $\mathfrak{M}$. In light of Theorem 2.3 and the fact (essentially due to Ehrenfeucht and Jensen, see [Sm, Theorem 5.10]) that any two countable elementarily equivalent recursively saturated models of $Z F$ with the same standard system are isomorphic, it suffices to show that

(1) $\operatorname{SSy}(\mathfrak{M})=\operatorname{SSy}(\mathfrak{M}[G])$, and

(2) recursive saturation is preserved under set generic extensions.

To see that (1) holds first note that no element of $\mathfrak{M}$ gains a new member in $\mathfrak{M}[G]$, since even in the case of nonstandard models generic extensions are end extensions $([\mathrm{Be}],[\mathrm{MD}])$. So, in particular, $\omega^{\mathfrak{M}}=\omega^{\mathfrak{M}[G]}$. But each member of the standard system of a model is arithmetically coded by some nonstandard integer of the same model. Therefore (1) holds because $\mathfrak{M}$ and $\mathfrak{M}[G]$ have the same nonstandard integers.

To see that recursive saturation is preserved under set generic extensions let us first recall the following elegant characterization of recursive saturation of models of ZF.

Lemma 2.6.1 (Schlipf [Sc]). A model $\mathfrak{M}$ of $Z F$ is recursively saturated iff

(a) $\mathfrak{M}$ is not $\omega$-standard, and

(b) $\left\{\alpha \in \operatorname{Ord}(\mathfrak{M}): V_{\alpha}^{\mathfrak{M}} \prec \mathfrak{M}\right\}=S$ is unbounded in $\operatorname{Ord}(\mathfrak{M})$.

In light of Lemma 2.6.1, and the fact that the property of not being $\omega$-standard is preserved in any extension of $\mathfrak{M}$, we just need to verify that property (b) is preserved under set generic extensions. Fix an ordinal $\gamma \in S$ such that $\mathbb{P} \in V_{\gamma}^{\mathfrak{M}}$. Clearly if $\alpha \geq \gamma$ and $\alpha \in S$ then $G$ is $\mathbb{P}$-generic over $V_{\alpha}^{\mathfrak{M}}$ 
and $V_{\alpha}^{\mathfrak{M}}[G]=V_{\alpha}^{\mathfrak{M}[G]}$. We claim:

$$
\forall \alpha \in S\left(\gamma \leq \alpha \Rightarrow V_{\alpha}^{\mathfrak{M}}[G] \prec \mathfrak{M}[G]\right)
$$

To prove $(*)$ let $\varphi(\mathbf{m})$ denote a first order formula in the language $\{\in\}$ with parameters $\mathbf{m}$ from $V_{\alpha}^{\mathfrak{M}}[G]$. Suppose, furthermore, that

$$
V_{\alpha}^{\mathfrak{M}}[G] \vDash \varphi(\mathbf{m}) .
$$

Clearly, for some $p \in G$, we have

$$
V_{\alpha}^{\mathfrak{M}} \vDash " p \Vdash \varphi(\mathbf{m}) ",
$$

which coupled with $V_{\alpha}^{\mathfrak{M}} \prec \mathfrak{M}$ implies

$$
\mathfrak{M} \vDash " p \Vdash \varphi(\mathbf{m}) ",
$$

yielding in turn

$$
\mathfrak{M}[G] \vDash \varphi(\mathbf{m}) .
$$

This establishes $(*)$. So property (b) is also preserved by set generic extensions as well. This concludes the proof of Theorem 2.6.

3. Counting standard models. The proof of our first result is inspired by the proof of Morley's classical theorem [Mor] stating that a countable theory $T$ in $L_{\omega_{1}, \omega}$ with more than $\aleph_{1}$ nonisomorphic models must already have $2^{\aleph_{0}}$ models. Morley's proof exploits the machinery of Scott sentences, which we briefly review here. We recommend $[\mathrm{Ba}]$ or $[\mathrm{Ke}]$ for reference on Scott sentences. Let $\tau$ denote a fixed countable signature and $\mathfrak{M}$ denote a model of signature $\tau$. One first looks at the equivalence relation $\equiv^{\alpha}$, where $\alpha$ is an ordinal, defined on finite sequences $\mathbf{a}$ and $\mathbf{b}$ of the same length from $\mathfrak{M}$ by: $\mathbf{a} \equiv{ }^{\alpha} \mathbf{b}$ iff for every formula $\varphi(\mathbf{x})$ of $L_{\omega_{1}, \omega}$ of quantifier rank less than or equal to $\alpha$,

$$
(\mathfrak{M}, \mathbf{a}) \vDash \varphi(\mathbf{a}) \quad \text { iff } \quad(\mathfrak{M}, \mathbf{b}) \vDash \varphi(\mathbf{b}) .
$$

This turns out to be equivalent to the following inductive algebraic definition:

- $\mathbf{a} \equiv{ }^{0} \mathbf{b}$ iff for all atomic formulas $\varphi(\mathbf{x}),(\mathfrak{M}, \mathbf{a}) \vDash \varphi(\mathbf{a})$ iff $(\mathfrak{M}, \mathbf{b}) \vDash \varphi(\mathbf{b})$.

- $\mathbf{a} \equiv^{\alpha+1} \mathbf{b}$ iff $\forall c \exists d\left((\mathbf{a}, c) \equiv^{\alpha}(\mathbf{b}, d)\right)$ and $\forall d \exists c\left((\mathbf{a}, c) \equiv^{\alpha}(\mathbf{b}, d)\right)$.

- $\mathbf{a} \equiv{ }^{\alpha} \mathbf{b}$ iff $\forall \beta<\alpha\left(\mathbf{a} \equiv{ }^{\alpha} \mathbf{b}\right)$, where $\alpha$ is a limit ordinal.

The Scott rank of a model $\mathfrak{M}$, denoted by $\operatorname{sr}(\mathfrak{M})$, is the first ordinal $\delta$ such that for all finite sequences $\mathbf{a}$ and $\mathbf{b}$ from $\mathfrak{M}, \mathbf{a} \equiv{ }^{\delta} \mathbf{b} \Rightarrow \mathbf{a} \equiv^{\delta+1} \mathbf{b}$. Scott observed that $\operatorname{sr}(\mathfrak{M})<|\mathfrak{M}|^{+}$and he showed that this implies that for a countable model $\mathfrak{M}$ the isomorphism type of $\mathfrak{M}$ is characterized by a single $L_{\omega_{1}, \omega}(\tau)$ sentence $\sigma^{\mathfrak{M}}$ among countable models, often known as a Scott sentence of $\mathfrak{M}$.

We are now ready to state Morley's theorem. 
MORLEY'S THEOREM [Mor]. If $T$ is a countable theory in $L_{\omega_{1}, \omega}(\tau)$ having fewer than continuum many nonisomorphic countable models, then for each countable ordinal $\alpha, T$ has at most countably many nonisomorphic models of Scott rank $\leq \alpha$.

Now suppose that $\alpha$ is a countable ordinal and that $T$ is some completion of ZF; we are interested in the number $\mu(T, \alpha)$ of transitive models of $T$ of height $\alpha$. Let $\sigma^{\alpha}$ denote a Scott sentence of the structure $(\alpha, \in)$, and consider the new sentence $\sigma^{\alpha}$ obtained by restricting all quantifiers of $\sigma^{\alpha}$ to the class Ord of ordinals. Clearly, for any model $\mathfrak{M}$ of ZF, we have:

$$
\mathfrak{M} \vDash \underline{\sigma}^{\alpha} \quad \text { iff } \quad \operatorname{Ord}(\mathfrak{M}) \cong(\alpha, \in) .
$$

Consequently,

$$
\mathcal{M}(T, \alpha)=\left\{\mathfrak{M}: \mathfrak{M} \text { is a countable model of } T \cup\left\{\underline{\sigma}^{\alpha}\right\}\right\} .
$$

Therefore by Morley's theorem if $\mu(T, \alpha)>\aleph_{1}$ then $\mu(T, \alpha)=2^{\aleph_{0}}$. But with a little more work we can do better, as witnessed by the following theorem:

Theorem 3.1. If $\mu(T, \alpha)>\aleph_{0}$ then $\mu(T, \alpha)=2^{\aleph_{0}}$. Indeed, the result is true for any countable theory $T$ containing the axiom " $\in$ is extensional", where $\mu(T, \alpha)$ is the number of countable transitive models $M$ of $T$ with $\varrho(M)=\alpha$.

Proof. If, on the contrary, $\mu(T, \alpha)<2^{\aleph_{0}}$ then Morley's theorem assures us that $\mu(T, \alpha) \leq \aleph_{0}$ once we have the following lemma.

Lemma 3.1.1. $\operatorname{sr}(M, \in) \leq \varrho(M)+1$ for each transitive set $M$.

Proof. Clearly it suffices to prove that for any two sequences $\left(a_{1}, \ldots, a_{n}\right)$ and $\left(b_{1}, \ldots, b_{n}\right)$ of elements of $M$,

(*) $\quad$ if $\left(a_{1}, \ldots, a_{n}\right) \equiv^{\varrho(M)+1}\left(b_{1}, \ldots, b_{n}\right)$ then $a_{i}=b_{i}$ for each $1 \leq i \leq n$.

But to prove $(*)$, in turn, it suffices to prove:

$(* *) \quad$ if $\{a, b\} \subseteq M$ and $\delta=\max \{\varrho(a), \varrho(b)\}$, then $a \equiv{ }^{\delta+1} b$ implies $a=b$.

We shall use induction on $\delta$ to prove $(* *)$. The case $\delta=0$ is trivial. For the inductive step, suppose on the contrary that $a \equiv^{\delta+1} b$ for some distinct elements $a$ and $b$ of $M$. Without loss of generality we can choose an element $c$ in $a \backslash b$. By transitivity of $M, c \in M$, so there must exist an element $d$ such that $(a, c) \equiv^{\delta}(b, d)$. This implies that $d \in b$, as well as $c \equiv^{\delta} d$. Since the ranks of $c$ and $d$ are $<\delta$, by our inductive assumption $c=d$. But then $c$ must be in $b$, contradicting our choice of $c$.

We are now ready to address the number of isomorphism types of countable well-founded models of a prescribed completion of ZF: 
TheOrem 3.2. Assume that there exists an uncountable well-founded model of ZFC and let $I$ denote the set of cardinals $\omega \cup\left\{\aleph_{0}, \aleph_{1}, 2^{\aleph_{0}}\right\}$. I is precisely $\{\mu(T): T$ is a complete extension of $Z F\}$.

Proof. Note that if $\mu(T)>\aleph_{1}$ then for some $\alpha<\aleph_{1}, \mu(T, \alpha)>\aleph_{1}$. But then by Theorem 3.1, $\mu(T, \alpha)=2^{\aleph_{0}}$. This shows that $\mu(T) \in I$ for any completion $T$ of ZF. Therefore to finish the proof we need to show that for any cardinal $\kappa \in I$, there is a theory $T_{\kappa}$ with $\mu\left(T_{\kappa}\right)=\kappa$. We shall do this by distinguishing the following cases:

CASE (A): $\kappa=0$,

CASE (B): $\kappa$ is a nonzero natural number $n$,

CASE $(\mathrm{C}): \kappa=\aleph_{0}$,

CASE (D): $\kappa=\aleph_{1}$, and

$\operatorname{CASE}(\mathrm{E}): \kappa=2^{\aleph_{0}}$.

Case (A). This is easy, since by Gödel's incompleteness theorem the theory $\mathrm{ZF}+\neg \mathrm{Con}(\mathrm{ZF})$ is consistent, so we can choose $T_{0}$ to be any completion of $\mathrm{ZF}+\neg \mathrm{Con}(\mathrm{ZF})$. Note that no model of $T$ can be even $\omega$-standard.

CASE (B). We first need to prove an easy lemma:

Lemma 3.2.1. Suppose $M$ is an uncountable transitive model of ZFC. The collection $\left\{\alpha<\omega_{1}^{\mathbf{L}}: L_{\alpha} \vDash \operatorname{Th}\left(\mathbf{L}^{M}\right)\right\}$ is unbounded in $\omega_{1}^{\mathbf{L}}$.

Proof. Since $M$ is a model of $\mathrm{ZFC}, \operatorname{Ord}(M)$ and $M$ are equinumerous. Hence $\operatorname{Ord}(\mathfrak{M})=\gamma \geq \omega_{1}$. Therefore,

$$
\mathbf{L} \vDash \text { " } \gamma \geq \omega_{1} \text { and } L_{\gamma} \text { is a model of ZFC". }
$$

Now argue in $\mathbf{L}$. By a routine Löwenheim-Skolem argument, for any countable ordinal $\alpha$ there exists a countable elementary submodel $\mathfrak{N}$ of $L_{\gamma}$ with $\alpha \subseteq \operatorname{Ord}(\mathfrak{N})$. By Gödel's condensation lemma this implies that the transitive collapse of $\mathfrak{N}$ is some $L_{\beta}$, where $\alpha<\beta<\omega_{1}$.

Given any nonzero natural number $n$, define the ordinal $\gamma_{n}$ to be the first ordinal $\gamma$ such that:

(1) $L_{\gamma} \vDash \mathrm{ZF}$, and

(2) there are at least $n-1$ transitive models of $\mathrm{ZF}+\mathbf{V}=\mathbf{L}$ of height less than $\gamma$ which are elementarily equivalent to $L_{\gamma}$.

The existence and countability of $\gamma_{n}$ is an immediate consequence of Lemma 3.2.1. Note that $L_{\gamma_{1}}$ is the so-called Shepherdson-Cohen minimal model of set theory. Let $T_{n}=\operatorname{Th}\left(L_{\gamma_{n}}\right)$. To show that $\mu\left(T_{n}\right)=n$ note that by the very definition of $\gamma_{n}, \mu\left(T_{n}\right) \geq n$. It is easy to see that if $\mu\left(T_{n}\right)>n$, then there is an ordinal $\beta$ such that $\beta>\gamma_{n}$ with $L_{\beta} \equiv L_{\gamma_{n}}$. But then

$$
L_{\beta} \vDash " \operatorname{Th}\left(L_{\gamma_{n}}\right) \text { has at least } n \text { transitive models", }
$$


which implies

$L_{\beta} \vDash$ "there exist distinct $\alpha_{1}, \ldots, \alpha_{n}$ with $L_{\alpha_{1}} \vDash \mathrm{ZF}$ and $\bigwedge_{i \leq n} L_{\alpha_{1}} \equiv L_{\alpha_{i}}$ ", but since $L_{\beta} \equiv L_{\gamma_{\kappa}}$,

$L_{\gamma_{\kappa}} \vDash$ "there exist distinct $\alpha_{1}, \ldots, \alpha_{n}$ with $L_{\alpha_{1}} \vDash \mathrm{ZF}$ and $\bigwedge_{i \leq n} L_{\alpha_{1}} \equiv L_{\alpha_{i}}$ ", contradicting the minimality $\gamma_{n}$.

Case (C). Similar to Case (B), define $\gamma_{\omega}$ to be the first ordinal $\gamma$ such that

(1) $L_{\gamma} \vDash \mathrm{ZF}$, and

(2) $\left\{\alpha<\gamma: L_{\alpha} \equiv L_{\gamma}\right\}$ is infinite.

The existence and countability of $\gamma_{\omega}$ is an immediate consequence of Lemma 3.2.1. Let $T_{\aleph_{0}}=\operatorname{Th}\left(L_{\gamma_{\omega}}\right)$. Clearly $\mu\left(T_{\aleph_{0}}\right) \geq \aleph_{0}$. It is easy to see that the height of no transitive model of $\operatorname{Th}\left(L_{\gamma_{\omega}}, \in\right)$ can be greater than $\gamma_{\omega}$, for if $\delta \geq \gamma_{\omega}$, and $L_{\delta}$ satisfies ZF, then in particular it can correctly compute the first order theory of models which are elements of $L_{\delta}$. Therefore

$$
L_{\delta} \vDash "\left\{\alpha<\gamma_{\omega}: L_{\alpha} \equiv L_{\gamma_{\omega}}\right\} \text { is infinite". }
$$

Consequently $L_{\delta}$ will satisfy the sentence $\varphi$, where

$$
\begin{aligned}
& \varphi=\text { "there exist distinct }\left(\alpha_{n}: n \in \omega\right) \\
& \text { with } L_{\alpha_{0}} \vDash \mathrm{ZF} \text { and } \forall n \in \omega\left(L_{\alpha_{0}} \equiv L_{\alpha_{n}}\right) " .
\end{aligned}
$$

The minimality of $\gamma_{\omega}$, on the other hand, implies that $\varphi$ is false in $L_{\gamma_{\omega}}$, in turn establishing that $\mu\left(T_{\aleph_{0}}\right)=\aleph_{0}$.

CASE (D). If $T$ is an extension of ZF $+\mathbf{V}=\mathbf{L}$, then $\mu(T) \leq \aleph_{1}$, thanks to Gödel's condensation lemma. To arrange a $T$ such that equality holds, let $T$ denote $\operatorname{Th}\left(\mathbf{L}^{M}\right)$, where $\mathfrak{M}$ is an uncountable model of ZFC. By an easy Löwenheim-Skolem reasoning similar to the proof of Lemma 3.2.1, $\mu(T)$ is precisely $\aleph_{1}$.

CAse (E). Let $\mathbb{P}$ denote Cohen's partial order for adding a generic real. $\mathbb{P}$ is easily seen to be weakly homogeneous and perfect. Therefore, by using Theorems 2.4 and 2.5, we can start with any countable standard model of ZF and force with $\mathbb{P}$ to obtain $2^{\aleph_{0}}$ distinct transitive models, any two of which are elementarily equivalent (for a more general version of this phenomenon, see Theorem 3.5). This concludes the proof of Theorem 3.2.

REMARK 3.3. Theorem 3.2 remains valid if $\mathrm{ZF}$ is replaced with the Kripke-Platek set theory KP, or at the other extreme, if ZF is replaced with the Kelley-Morse theory of classes KM, provided $\mu(T)$ is interpreted in the 
latter case as counting the number of the countable transitive $\beta$-models of a completion $T$ of $\mathrm{KM}$.

We now concentrate on the behavior of $\mu(T, \alpha)$. By Gödel's condensation lemma we know that if any extension $T$ of $Z F$ includes the axiom $\mathbf{V}=\mathbf{L}$ then $\mu(T, \alpha) \leq 1$. The converse fails in a canonical manner, as witnessed by the following classical theorem of Solovay.

TheOrem 3.4 (Solovay). Assume $\alpha$ is a countable ordinal such that $L_{\alpha} \vDash Z F$. There exists a complete theory $T$ extending $Z F+\mathbf{V} \neq \mathbf{L}$ such that $\mu(T, \alpha)=1$.

Proof. This is an immediate consequence of Solovay's construction [JS] of a constructible partial order $\mathbb{P}$ such that

$$
1_{\mathbb{P}} \Vdash " \mathbf{V}=\mathbf{L}(G) \text { and } G \text { is a nonconstructible } \Delta_{3}^{1} \text { real". }
$$

The next result generalizes a theorem of H. Friedman [Fr-1, Theorem 6.4] who showed that every countable transitive model of $\mathrm{ZF}+\mathbf{V} \neq \mathbf{O D}$ is elementarily equivalent to another transitive model of the same height, that is, if $T$ is a completion of $\mathbf{Z F}+\mathbf{V} \neq \mathbf{O D}$ with $\mu(T, \alpha) \geq 1$ then $\mu(T, \alpha) \geq 2$. Our proof uses an omitting types argument, and is simpler than Friedman's, which used methods from infinitary logic.

TheOREm 3.5. If $\alpha<\omega_{1}, T$ is a completion of $Z F+\mathbf{V} \neq \mathbf{O D}$, and $\mu(T, \alpha) \geq 1$, then $\mu(T, \alpha)=2^{\aleph_{0}}$.

Proof. Suppose $M$ is a countable transitive model of $T$ of height $\alpha$ and let $T^{*}=\operatorname{Th}(M, \in, \beta)_{\beta \in \alpha}$ (so $T^{*}$ is a theory formulated in the language consisting of $\in$ and a constant symbol for each ordinal of $M$ ). We shall use transfinite induction on $\gamma<\omega_{1}$ to build a sequence of distinct transitive models $\left(M_{\gamma}: \gamma<\omega_{1}\right)$ of height $\alpha$ of $T^{*}$ with $M_{0}=M$. Since distinct transitive sets are nonisomorphic this will immediately imply that $\mu(T, \alpha)$ $=2^{\aleph_{0}}$, thanks to Theorem 3.1.

To construct $M_{1}$ we observe that there exists $s_{0} \subseteq \alpha$ such that $s_{0} \in$ $M \backslash$ HOD $^{M}$. This is an immediate consequence of (1) and (2) below:

(1) By a result of Vopěnka and Balcar [J, Lemma 15.1] if $M$ and $N$ are transitive models of $\mathrm{ZF}$ of the same height which have the same sets of ordinals, and at least one of $M$ or $N$ satisfies AC, then $M=N$ (this fact is often stated and proved for inner models, but the proof establishes the more general result).

(2) $\mathbf{H O D}^{M} \neq M$, and the axiom of choice holds in $\mathbf{H O D}^{M}$.

Now consider the 1-types $\Pi(x)$ and $\Lambda_{0}(x)$ defined by

$$
\begin{aligned}
\Pi(x) & =\{\text { " } x \in \text { Ord" }\} \cup\{x \neq \beta: \beta \in \alpha\}, \\
\Lambda_{0}(x) & =\{\text { " } x \subseteq \text { Ord" }\} \cup\left\{\beta \in x: \beta \in s_{0}\right\} \cup\left\{\beta \notin x: \beta \notin s_{0}\right\} .
\end{aligned}
$$


Clearly $\Pi(x)$ is locally omitted by $T^{*}$ since $M_{0}$ omits $\Pi(x)$. To see that $\Lambda_{0}(x)$ is also locally omitted by $T^{*}$, suppose that for some formula $\psi(x, \mathbf{m})$, where $\mathbf{m}$ denotes a finite sequence of ordinal parameters less than $\alpha$, we have

$$
T^{*} \vdash \exists x(\psi(x, \mathbf{m}))
$$

and

$$
\beta \in s_{1} \quad \text { iff } \quad T^{*} \vdash(\psi(x, \mathbf{m}) \rightarrow \beta \in x)
$$

But then "the unique $x$ satisfying $\psi(x, \mathbf{m})$ " serves as a definition of $s_{0}$ in $M_{0}$, contradicting the choice of $s_{0} \notin \mathbf{H O D}^{M}$. By the omitting types theorem [CK, Theorems 2.2.9 and 2.2.15] there exists a countable model $M_{1}$ of $T^{*}$ which omits both types $\Pi$ and $\Lambda_{0}$. Since $\operatorname{Ord}\left(M_{1}\right)=\alpha, M_{1}$ is well-founded and can be assumed to be transitive. Moreover, since $s_{0} \notin M_{0}, M_{0} \neq M$. We can now easily repeat this process $\omega_{1}$ times. For suppose we have constructed distinct transitive models $\left(M_{\gamma}: \gamma<\delta\right)$ for some $\delta<\omega_{1}$ such that each $M_{\gamma}$ is a transitive model of $T^{*}$ of height $\alpha$. For each $\gamma<\delta$, choose $s_{\gamma} \subseteq \alpha$ such that $s_{\gamma} \in M_{\gamma} \backslash \mathbf{H O D}^{M_{\gamma}}$ and let $\Lambda_{\gamma}(x)$ denote the type defined by

$$
\Lambda_{\gamma}(x)=\{\text { " } x \subseteq \text { Ord" }\} \cup\left\{\beta \in x: \beta \in s_{\gamma}\right\} \cup\left\{\beta \notin x: \beta \notin s_{\gamma}\right\} .
$$

Since each of the types $\Lambda_{\gamma}(x)$ is locally omitted by $T^{*}$ the omitting types theorem implies that there is a transitive model $M_{\delta}$ of $T^{*}$ omitting the type $\Pi(x)$ as well as the set of types $\left\{\Lambda_{\gamma}(x): \gamma<\delta\right\}$. Since $s_{\gamma} \in M_{\gamma} \backslash M_{\delta}$ for all $\gamma<\delta, M_{\delta} \neq M_{\gamma}$ for $\gamma<\delta$.

In light of the fact that $\mathrm{ZF}+\mathbf{V}=\mathbf{O D} \vdash \mathrm{AC}$ we have the following immediate corollary.

COROLlary 3.5.1. If $T$ is a complete extension of $Z F+\neg A C$, then $\mu(T, \alpha)=2^{\aleph_{0}}$.

We now investigate the behavior of $\mu(T, \alpha)$ when $T$ includes the axiom $\mathbf{V}=\mathbf{O D}$. Recall that if $T$ includes the axiom $\mathbf{V}=\mathbf{O D}$ then $T$ has definable Skolem functions and therefore $T$ has a pointwise definable model, i.e., a model each element of which is definable by a parameter free first order formula.

TheOREM 3.6. Suppose $T$ is a completion of $Z F+\mathbf{V}=\mathbf{O D}$ which has a well-founded model. If $\alpha$ is the height of the pointwise definable model of $T$ and $\mu(T, \alpha)>1$, then there is a nontrivial elementary embedding $j: L_{\alpha} \rightarrow L_{\alpha}$.

Proof. Suppose $M$ and $N$ are distinct transitive models of $T$ of height $\alpha$, where $M$ is the pointwise model of $T$. It is easy to see that every pointwise definable model of a complete theory is a prime model, i.e., it (uniquely) elementarily embeds into any model of its theory. So there is an elementary embedding $j: M \rightarrow N$. Since all elements of $M$ and $N$ are definable from 
their ordinals, $j$ does not map $\alpha$ onto $\alpha$ and therefore $j$ moves some ordinal. Now consider the restriction $j^{*}$ of $j$ to the constructible universe $\mathbf{L}^{M}$ of $M$. The constructible universe of any model of ZF is first order definable inside the model itself, which assures us that $j^{*}: \mathbf{L}^{M} \rightarrow \mathbf{L}^{N}$ is also an elementary embedding. But $M$ and $N$ are of the same height $\alpha$, so $\mathbf{L}^{M}=\mathbf{L}^{N}=L_{\alpha}$. Therefore $j^{*}$ is a nontrivial elementary embedding of $L_{\alpha}$ into itself.

REMARK 3.6.1. If $j: M \rightarrow M$ is a nontrivial elementary embedding of a transitive model $M$ of ZFC into itself and $\theta$ is the first ordinal moved by $j$ then

- $M \vDash$ " $\theta$ is completely ineffable" (Kleinberg [Kl]), and

- $M \vDash " \forall m, n<\omega\left(\theta\right.$ is $\Pi_{n}^{m}$-indescribable)" (Reinhardt-Silver [Ka, 9.8]).

Therefore if $T$ is a completion of $\mathrm{ZF}+\mathbf{V}=\mathbf{O D}$ such that $\mu(T, \alpha)>1$ and $\alpha$ is the height of the pointwise definable model of $T$, then by Theorem 3.6, $L_{\alpha}$ must contain a completely ineffable, totally indescribable cardinal.

Since pointwise definable models lack nontrivial elementary self-embeddings, Theorems 3.5 and 3.6 yield the following corollary.

Corollary 3.6.2. If $\left(L_{\alpha}, \in\right)$ is pointwise definable then for any completion $T$ of $Z F$ :

(a) If $T \vdash \mathbf{V}=\mathbf{O D}$ then $\mu(T, \alpha) \in\{0,1\}$.

(b) If $T \vdash \mathbf{V} \neq \mathbf{O D}$ then $\mu(T, \alpha) \in\left\{0,2^{\aleph_{0}}\right\}$.

Recall that the Shepherdson-Cohen minimal model is pointwise definable [C, p. 105]. Theorem 3.7 below shows that under a mild assumption there are many ordinals $\alpha$ such that $L_{\alpha}$ is a pointwise definable model of ZF.

THEOREM 3.7. Assume that there is an uncountable well-founded model of ZFC. Then there are arbitrarily large ordinals $\alpha<\omega_{1}^{\mathbf{L}}$ such that $\left(L_{\alpha}, \in\right)$ is a pointwise definable model of $Z F$.

Proof. We first note that if $\alpha$ is an ordinal with the property that $L_{\alpha}$ is pointwise definable then $L_{\alpha}$ is also pointwise definable in the sense of the constructible universe because the satisfaction predicate for set models is a $\Delta_{1}$-predicate in the Lévy hierarchy, and hence absolute for transitive models. This immediately implies that $\alpha<\omega_{1}^{\mathbf{L}}$ since $\alpha \subseteq L_{\alpha}$. Now consider the set

$$
S=\left\{\alpha<\omega_{1}^{\mathbf{L}}: L_{\alpha} \text { is a pointwise definable model of } \mathrm{ZF}\right\} .
$$

Suppose on the contrary that $S$ is bounded in $\omega_{1}^{\mathbf{L}}$ and let $\beta=\sup S$. Since $\beta$ is countable in $\mathbf{L}$, by the reflection theorem there exists some ordinal $\gamma$ such that $\beta$ is countable in $L_{\gamma}$, therefore by a routine Löwenheim-Skolem 
argument there is some countable $M \prec L_{\gamma}$ with $\beta \subseteq M$. By Gödel's condensation lemma the transitive collapse of $M$ is isomorphic to some $L_{\bar{\gamma}}$ with $\bar{\gamma}<\omega_{1}^{\mathrm{L}}$. Note that $\beta$ is countable in every $L_{\theta}$ as soon as $\bar{\gamma} \leq \theta$. We can now invoke Lemma 3.2.1 to choose an ordinal $\theta$ such that $\bar{\gamma} \leq \theta<\omega_{1}^{\mathrm{L}}$, with $L_{\theta} \vDash \mathrm{ZF}$.

The key observation is that $\beta$ is definable in $L_{\theta}$ since the external definition of $\beta$ as the supremum of $S$ can serve to define $\beta$ in $L_{\theta}$ as well. Therefore $\beta$ is a member of the elementary submodel $D$ of $L_{\theta}$ consisting of the pointwise definable elements of $L_{\theta}$. Since $\beta$ is countable in $D$ there is some surjection $f: \omega \rightarrow \beta$ with $f \in D$. So $f(n) \in D$ for each $n \in \omega$ (since $\omega \subseteq D$ ) and therefore $\beta \subseteq D$. But then the transitive collapse of $D$ is equal to $L_{\eta}$ for some $\eta>\beta$, contradicting the choice of $\beta$. Hence $S$ is unbounded in $\omega_{1}^{\mathrm{L}}$, as advertised.

We now show that it is possible to have $\mu(T, \alpha)=2^{\aleph_{0}}$ for completions $T$ of $\mathrm{ZF}+\mathbf{V}=\mathbf{O D}$. In light of Remark 3.6.1, it is not surprising that large cardinals are involved in parts (a) and (b).

THEOREM 3.8. If $\alpha<\omega_{1}$ and $T$ is a completion of $Z F+\mathbf{V}=\mathbf{O D}$ satisfying at least one of the conditions (a) or (b) below, then $\mu(T, \alpha) \in$ $\left\{0,2^{\aleph_{0}}\right\}$.

(a) $T \vdash$ "there is a precipitous ideal on $\aleph_{1}$ ".

(b) [Woodin, private communication] $T \vdash$ "there is a proper class of Ramsey cardinals".

Proof. (a) Suppose $M$ is a countable transitive model of $\mathrm{ZF}+\mathbf{V}=\mathbf{O D}$ in which there exists a precipitous ideal on $\aleph_{1}$, i.e., an ideal $I$ on $\aleph_{1}$ with the property

if $\mathbb{P}=\mathcal{P}\left(\aleph_{1}\right) / I$ and $G$ is $\mathbb{P}$-generic then $1_{\mathbb{P}} \Vdash$ " $V^{\aleph_{1}} / G$ is well-founded".

Use Theorem 2.4 to obtain a family of mutually generic filters $\left(G_{f}\right.$ : $\left.f \in 2^{\omega}\right)$ over the partial order $\mathbb{P}=\mathcal{P}\left(\aleph_{1}\right) / I$. By the very definition of being precipitous, $M^{\aleph_{1}} / G_{f}$ is well-founded in the sense of $M\left[G_{f}\right]$, and therefore really well-founded for each $f \in 2^{\omega}$. Let $N_{f}$ denote the transitive collapse of the generic ultrapower $M^{\aleph_{1}} / G_{f}$, which can be computed within the generic extension $M\left[G_{f}\right]$. To show that $N_{f}$ and $N_{g}$ are distinct when $f \neq g$, we prove a lemma:

LemmA 3.8.1. If $G$ and $\bar{G}$ are $\mathbb{P}$-generic filters over $M$ which are mutually generic then the ultrapowers $M^{\aleph_{1}} / G$ and $M^{\aleph_{1}} / \bar{G}$ are not isomorphic.

Proof. Let $N$ and $\bar{N}$ respectively denote the transitive collapses of $M^{\aleph_{1}} / G$ and $M^{\aleph_{1}} / \bar{G}$. We wish to show that $N \neq \bar{N}$. The crucial observation is that $\aleph_{1}^{M}$ is collapsed to a countable ordinal in $M^{\aleph_{1}} / G$ because $\aleph_{1}^{M}$ is the first ordinal moved by the elementary embedding $j: M \rightarrow N$. Hence 
there is a surjective map $\chi: \omega \rightarrow \aleph_{1}^{M}$ in $N$. Since $N$ and $\bar{N}$ can be computed respectively within $M[G]$ and $M[\bar{G}], N \subseteq M[G]$ and $\bar{N} \subseteq M[\bar{G}]$. By Theorem 2.7, $M[G] \cap M[\bar{G}]=M$, hence $\chi \in N \backslash \bar{N}$. Therefore $N \neq \bar{N}$.

Note that for each $f \in 2^{\omega}, \operatorname{Ord}\left(N_{f}\right)=\operatorname{Ord}(M)$ since the $\in$-relation on the well-founded ultrapower $M^{\aleph_{1}} / G_{f}$ is well-founded and set-like in the sense of $M\left[G_{f}\right]$. By the uniqueness condition in Mostowski's collapsing lemma the $N_{f}$ 's are nonisomorphic and yet, as ultrapowers of $M$, they all satisfy the same first order theory $\operatorname{Th}(M)$. This concludes the proof of part (a) of Theorem 3.8.

(b) This follows from the machinery of stationary tower forcing introduced by Woodin ([W-1], [W-2, Sec. 2.3]). The full stationary tower is a class notion of forcing $\mathbb{P}_{\infty}=\bigcup_{\alpha \in \text { Ord }} \mathbb{P}_{<\alpha}$, where the elements of $\mathbb{P}_{<\alpha}$ consist of the so-called stationary elements in $V_{\alpha}$, i.e., nonempty elements $a$ of $V_{\alpha}$ which have the property that for any function $f:(\bigcup a)^{<\omega} \rightarrow \bigcup a$ there is $b \in a$ such that $f\left[b^{<\omega}\right] \subseteq b$. The partial ordering of $\mathbb{P}_{<\alpha}$ is given by

$$
a \leq b \quad \text { iff } \quad\{s \cap(\bigcup b): s \in a\} \subseteq b .
$$

Woodin [W-1, Theorem 1] proved:

- If $G$ is a $\mathbb{P}_{\infty}$-generic filter over a countable transitive model $M$ of ZFC then there is an elementary embedding $j: M \rightarrow\left(V^{\mathbb{P}_{\infty}} / G\right)^{M[G]}$, where $V^{\mathbb{P}_{\infty}} / G$ is an appropriate limit ultrapower computed in $V[G]$. Moreover, if $M$ has a proper class of Ramsey cardinals then $M[G] \cong\left(V^{\mathbb{P}_{\infty}} / G\right)^{M[G]}$ (Woodin's proof actually works with the weaker hypothesis "there is a proper class of completely Jónsson cardinals").

Since it is known that $\mathbb{P}_{\infty}$ absorbs any set forcing, by Theorem 2.5 there are continuum many distinct $M[G]$ 's, which shows that for $T=\operatorname{Th}(M)$ and $\alpha=$ the height of $M, \mu(T, \alpha)=2^{\aleph_{0}}$. The fact that $M$ can be assumed to satisfy $\mathbf{V}=\mathbf{O D}$ is the consequence of a result of Jensen stated in [Mc, Sec. 5].

REMARK 3.8.1. The work of Jech-Magidor-Mitchell-Prikry [JMMP] shows (among other things) that the Lévy collapse which transforms a measurable cardinal to $\aleph_{1}$ produces a precipitous ideal on $\aleph_{1}$. Since Lévy's notion of forcing is weakly homogeneous, the generic extension satisfies $\mathbf{V} \neq \mathbf{O D}$. However, one can always force $\mathbf{V}=\mathbf{O D}$ without perturbing a prescribed initial segment of the universe, thanks to a theorem of Roguski [Ro] which states that if $M$ is a countable transitive model of $\mathrm{ZF}$ and $\alpha$ is an ordinal of $M$ then there is a generic extension $N$ of $M$ in which $\mathbf{V}=\mathbf{O D}$ holds and such that $V_{\alpha}^{M}=V_{\alpha}^{N}$. Therefore the theory ZF $+\mathbf{V}=\mathbf{O D}+$ "there is a precipitous ideal on $\aleph_{1}$ " is consistent relative to $\mathrm{ZF}+$ "there exists a measurable cardinal". 
Theorem 3.9 [Kunen, private communication]. If for some $\beta>\alpha$ we have $\left(L_{\beta}, \alpha\right) \vDash$ " $\alpha$ is an inaccessible cardinal" and $L_{\beta} \vDash Z F$, then there is some completion $T$ of $Z F+\mathbf{V}=\mathbf{O D}$ such that $\mu(T, \alpha) \in\left\{0,2^{\aleph_{0}}\right\}$.

Proof. Suppose $\beta>\alpha$, and $\left(L_{\beta}, \alpha\right) \vDash$ " $\alpha$ is inaccessible". Use McAloon's techniques [Mc] to arrange a cardinal preserving extension $M$ of $L_{\beta}$ such that

$$
M \vDash " 2^{\aleph_{0}}>\aleph_{1} \text { and } V_{\alpha} \text { is a model of } \mathbf{V}=\mathbf{O D} \text { ". }
$$

Let $T=\operatorname{Th}\left(V_{\alpha}^{M}\right)$. Since the universe constructed from a real satisfies $\mathrm{CH}$, $L_{\beta}(T)$ satisfies $\mathrm{CH}$. Moreover, since $M$ is a cardinal preserving extension of $L_{\beta}, \aleph_{1}^{M}=\aleph_{1}^{L_{\beta}(T)}=\aleph_{1}^{L_{\alpha}}<\alpha$, which implies that within $L_{\beta}(T)$ every model of ZFC of height $\alpha$ must satisfy $\mathrm{CH}$ as well, so

$$
\left(L_{\beta}(T), \alpha\right) \vDash \text { "there is no standard model of } T \text { of height } \alpha \text { ". }
$$

However, if $N$ is any forcing extension of $L_{\beta}(T)$ obtained by making $\alpha$ countable in the standard way then $N$ will contain a model of height $\alpha$, by Mostowski's absoluteness theorem for $\Pi_{1}^{1}$ statements [Mi, Theorem 17.5]. By Theorem 2.5 there are $2^{\aleph_{0}}$ distinct extensions of this form, which implies that there must be $2^{\aleph_{0}}$ distinct models of $T$ of height $\alpha$.

REMARK 3.10. (a) Note that as soon as $T$ includes the axiom "there exists a measurable cardinal" and $\mu(T, \alpha) \neq 0$, then $\mu(T, \alpha) \geq \aleph_{0}$. Assuming $T$ includes AC this easily follows by looking at the transitive collapses of the iterated ultrapowers of any transitive model of $T$ of height $\alpha$. On the other hand, if $T$ does not include AC, then we are done by Corollary 3.5.1.

(b) There is a fundamental difference between Theorem 3.8 and Theorem 3.9. In Theorem 3.9 the theory $T$ can be arranged to have the peculiar property that $\mu(T, \alpha)=2^{\aleph_{0}}$ but $\mu\left(T, \alpha_{T}\right)=1$, where $\alpha_{T}$ is the height of the pointwise definable model of $T$. In light of Theorem 3.6 and Remark 3.6.1, in order to arrange $\mu\left(T, \alpha_{T}\right)=1$ we just need to choose the $\alpha$ and $\beta$ of Theorem 3.6.1 so that $L_{\beta} \vDash$ " $\alpha$ is the first inaccessible cardinal".

4. Counting nonstandard models. We now turn to the question of calculating the number $\mu(T, \tau)$ of isomorphism types of countable models of a completion $T$ of $\mathrm{ZF}$ whose ordered sets of ordinals have a prescribed order type $\tau$ that is not well-ordered. The key result of this section is Theorem 4.1 which, coupled with a key result of Harvey Friedman, limits $\mu(T, \tau)$ to only two values 0 and $2^{\aleph_{0}}$, even when $T$ includes the axiom $\mathbf{V}=\mathbf{L}$, in sharp contrast to the well-founded case (see Corollary 4.3). The following definitions play a central role in this section:

- A model $\mathfrak{N}$ is said to be an e.e.e. (elementary end extension) of $\mathfrak{M}$, written $\mathfrak{M} \prec \prec_{\mathrm{e}} \mathfrak{N}$, if $\mathfrak{M} \prec \mathfrak{N}$ and no member of $\mathfrak{M}$ gains a new member in the passage from $\mathfrak{M}$ to $\mathfrak{N}$. More precisely, $\mathfrak{M} \prec{ }_{\mathrm{e}} \mathfrak{N}$ if $\mathfrak{M}=(M, E)$ and 
$\mathfrak{N}=(N, F)$ and for all $a \in M, a_{E}=a_{F}$ (see the third clause of Definition 2.1 for $a_{E}$ ). If $\mathfrak{M} \prec_{\mathrm{e}} \mathfrak{N}$ we also refer to $\mathfrak{M}$ as an elementary initial segment of $\mathfrak{N}$.

- A subset $X$ of $M$ is a class of $\mathfrak{M}$ if $(\mathfrak{M}, X) \vDash \mathrm{ZF}(X)$, where $\mathrm{ZF}(X)$ is the natural extension of ZF in the language $\{\in, X\}$ in which the unary predicate $X$ is allowed to occur in the replacement scheme.

THEOREM 4.1. Every countable model of ZF has $2^{\aleph_{0}}$ countable nonisomorphic e.e.e.'s.

Proof. This is not too hard to prove for countable standard models, and more generally, if "nonisomorphic" is weakened to "nonisomorphic over $\mathfrak{M}$ ", where $\mathfrak{M}$ is the countable model to be end extended. But the proof of the nonstandard case requires considerably more work. Roughly speaking, in Lemmas 4.1.1 and 4.1.2 we first show by a forcing construction that there are a large number of dissimilar classes over any countable model $\mathfrak{M}$ of ZF and then, in Lemma 4.1.3, we employ a massive omitting types argument to show that each of these classes can be coded in an e.e.e. of $\mathfrak{M}$ from which $\mathfrak{M}$ itself can be unambiguously recovered.

We begin with a lemma concerning expansions of models of ZF which is inspired by a result of Simpson [Si] who used "Feferman forcing" to establish that any countable model $\mathfrak{M}$ of Peano arithmetic PA can be expanded to $(\mathfrak{M}, G)$, where $G$ is a unary predicate, such that $(\mathfrak{M}, G)$ is pointwise definable and satisfies $\mathrm{PA}(G)$ (i.e., the natural extension of $\mathrm{PA}$ in which formulas mentioning $G$ are allowed to appear in the induction scheme). As Simpson himself pointed out, the same technique works for models of ZFC, but since we are not assuming that $\mathfrak{M}$ satisfies the axiom of choice we cannot hope to be able to produce a $G$ as in Simpson's theorem since for such a $G,(\mathfrak{M}, G)$ satisfies $\mathbf{V}=\mathbf{O D}(G)$, which in turn implies that the axiom of choice holds in $\mathfrak{M}$. However, by modifying Simpson's strategy we can nevertheless ensure that all of the ordinals of $\mathfrak{M}$ are pointwise definable in some expansion $(\mathfrak{M}, G)$ satisfying $\mathrm{ZF}(G)$. On the other hand, it is known that every wellfounded model $M$ of $\mathrm{ZF}+\mathbf{V}=\mathbf{O D}$ of any cardinality has an undefinable class $X$ such that $M$ and $(M, X)$ have the same definable elements [E-2].

Lemma 4.1.1. Suppose $\mathfrak{M}$ is a countable model of ZF. There exists a class $G \subseteq \operatorname{Ord}(\mathfrak{M})$ such that each ordinal of $\mathfrak{M}$ is definable in $(\mathfrak{M}, G)$.

Proof. Consider the notion of forcing $\mathbb{P}=\left(2^{<\text {Ord }}\right)^{\mathfrak{M}}$ consisting of all functions $p$ in $\mathfrak{M}$ which map some ordinal of $\mathfrak{M}$ into $\{0,1\}$. It is well known that $(\mathfrak{M}, G) \vDash \mathrm{ZF}(G)$ (the published expositions of this fact in [Fe] and [Mos] work with models of ZFC, but the proof can be carried out without recourse to $\mathrm{AC})$. We now describe the construction of a sequence of conditions $\left(p_{n}\right.$ : $n \in \omega)$ giving rise to a generic $G$ to ensure that all the ordinals of $\mathfrak{M}$ are 
definable in $(\mathfrak{M}, G)$. Let us begin with a few definitions, understood to be carried out within $\mathfrak{M}$.

For a formula $\varphi(a, G)$ of the forcing language, $p \in \mathbb{P}$, and $\alpha \in \operatorname{Ord}(\mathfrak{M})$ :

- $|p|=$ the domain of $p$, referred to as the length of $p$,

- $p \frown \alpha=$ the condition of length $|p|+\alpha+1$, defined by

$$
(p \frown \alpha)(\nu)= \begin{cases}p(\nu) & \text { if } \nu<|p|, \\ 0 & \text { if }|p| \leq \nu<\alpha, \\ 1 & \text { if } \nu=\alpha,\end{cases}
$$

- $\beta_{\varphi(a, G)}(p)=\min \{|q|: q \supseteq p$ and $q \| \varphi(a, G)\}$, and

- $\beta_{\varphi(\cdot, G)}(p, \alpha)=\sup \left\{\beta_{\varphi(a, G)}(p): a \in V_{\alpha}\right\}\left(\beta_{\varphi(\cdot, G)}\right.$ is well defined, thanks to the schema of replacement).

Enumerate the ordinals of $\mathfrak{M}$ as $\left(\alpha_{n}: n \in \omega\right)$, and to simplify matters, let us take advantage of the availability of a definable coding of finite sequences to assume that each formula of the forcing language has at most one parameter from $\mathfrak{M}$. Moreover, enumerate all formulas of the forcing language $\left(\varphi_{n}\left(a_{n}, G\right): n \in \omega\right)$ in such a manner to ensure that

- the parameter $a_{n}$ of $\varphi_{n}\left(a_{n}, G\right)$ is in $V_{\theta_{n}}^{\mathfrak{M}}$, where $\theta_{n}=\max \left(\alpha_{i}: i \leq n\right)$.

Let $p_{0}=\emptyset \frown \alpha_{0}$ (i.e., the sequence of $\alpha_{0}$ 0's followed by a 1 ), and choose $p_{1}$ extending $p_{0}$ deciding $\varphi_{0}\left(a_{0}, G\right)$ and such that the length of $p_{1}$ is precisely equal to $\beta_{\varphi_{0}(\cdot, G)}\left(p_{0}, \alpha_{0}\right)$ (this can be done by elongating a minimal such condition, if necessary, since $\left.a_{0} \in V_{\alpha_{0}}^{\mathfrak{M}}\right)$. Next, define $p_{2}=p_{1} \frown \alpha_{1}$, and choose $p_{3}$ to extend $p_{2}$ which decides $\varphi_{1}\left(a_{1}, G\right)$ and has length precisely equal to $\beta_{\varphi_{1}(\cdot, G)}\left(p_{2}, \max \left(\alpha_{0}, \alpha_{1}\right)\right)$. More generally, for $n \geq 1$ :

- $p_{2 n+1}$ extends $p_{2 n}$, decides $\varphi_{n}\left(a_{n}, G\right)$, and has length $\beta_{\varphi_{n}(\cdot, G)}\left(p_{2 n}, \theta_{n}\right)$;

- $p_{2 n}=p_{2 n-1} \frown \alpha_{n}$.

Clearly every sentence of the forcing language is decided by some $p_{n}$ and therefore $G=\bigcup_{n \in \omega} p_{n}$ is $\mathbb{P}$-generic over $\mathfrak{M}$. A straightforward induction argument shows that every $\alpha_{n}$ is definable in $(\mathfrak{M}, G)$, essentially because $\alpha_{0}$ can be read off from $G$ as the length of the longest initial segment of 0's of $G$, thereby rendering $\beta_{\varphi_{0}(\cdot, G)}\left(p_{0}, \alpha_{0}\right)$ definable from $G$, which allows $\alpha_{1}$ to be read off from $G$ as the length of the longest sequence of 0 's of the sequence succeeding the first $\beta_{\varphi_{0}(\cdot, G)}\left(p_{0}, \alpha_{0}\right)$ entries of $G$, in turn showing that $\beta_{\varphi_{1}(\cdot, G)}\left(p_{1}, \theta_{1}\right)$ is definable from $G$, etc.

\section{Lemma 4.1.2. If $\mathfrak{M}$ is a countable model of $Z F$ then}

$$
\mid\{\operatorname{Th}(\mathfrak{M}, X): X \text { is a class of } \mathfrak{M}\} \mid=2^{\aleph_{0}} .
$$

Proof. Let $G$ be as in Lemma 4.1 .1 and use the same notion of forcing $\mathbb{P}$ used in Lemma 4.1.1 to force over $(\mathfrak{M}, G)$ to obtain a continuum of distinct generic objects $\left\{Y_{\alpha}: \alpha<2^{\aleph_{0}}\right\}$ with each $Y_{\alpha} \subseteq \operatorname{Ord}(\mathfrak{M})$. If $\alpha \neq \beta$ then 
$\left(\mathfrak{M}, G, Y_{\alpha}\right)$ and $\left(\mathfrak{M}, G, Y_{\beta}\right)$ are not elementarily equivalent, thanks to the pointwise definability of ordinals in $(\mathfrak{M}, G)$. To see this, assume without loss of generality that $\gamma \in Y_{\alpha} \backslash Y_{\beta}$, and suppose the formula $\psi(G, \cdot)$ defines $\gamma$ in $(\mathfrak{M}, G)$. Clearly:

$\left(\mathfrak{M}, G, Y_{\alpha}\right) \vDash$ "the ordinal satisfying $\psi(G, \cdot)$ is in $Y_{\alpha}$ ",

but

$$
\left(\mathfrak{M}, G, Y_{\beta}\right) \vDash \text { "the ordinal satisfying } \psi(G, \cdot) \text { is not in } Y_{\beta} \text { ". }
$$

Since we can code each $\left(G, Y_{\alpha}\right)$ as a subset $X_{\alpha}$ of $\operatorname{Ord}(\mathfrak{M})$, we may conclude that $\operatorname{Th}\left(\mathfrak{M}, X_{\alpha}\right) \neq \operatorname{Th}\left(\mathfrak{M}, X_{\beta}\right)$ for $\alpha \neq \beta$.

The next lemma finetunes the classical result of Keisler-Morley on the existence of elementary end extensions of models of ZF ([KM], [CK, Theorem 2.2.18]) and is inspired by a result of Kossak and Paris [KP, Theorem 3.4] regarding models of Peano arithmetic whose exact analogue for models of ZF can be carried out only for models satisfying $\exists x(\mathbf{V}=\mathbf{O D}(x))$. Here we are not even assuming that local choice is necessarily available, but we can take advantage of the manageable global prewellordering afforded by the $V_{\alpha}$ hierarchy to prove a useful analogue of the Kossak-Paris result which is sufficient for our purposes.

Lemma 4.1.3. Suppose $\mathfrak{M}=(M, E)$ is a countable model of $Z F$ and $X \subseteq \operatorname{Ord}(\mathfrak{M})$ is a class of $\mathfrak{M}$. There is an e.e.e. $\mathfrak{N}=(N, F)$ of $\mathfrak{M}$ such that:

(a) $c_{F} \cap M=X$ for some $c \in N$, and

(b) $\mathfrak{M}$ is the largest proper elementary initial segment of $\mathfrak{N}$ (abbreviated as " $\mathfrak{N}$ is an e-minimal e.e.e. of $\mathfrak{M}$ ").

Proof. Suppose $X$ is a class of $M$, and for each $\alpha$ in $\operatorname{Ord}(\mathfrak{M})$, let $X \cap \alpha_{E}$ be coded by the element $a_{\alpha} \in M$, i.e., $X \cap \alpha=\left\{x \in M: x E a_{\alpha}\right\}$. Consider the following theory $T$ in the language consisting of $\in$ and constants for each member of $M$, and a new constant symbol $c$ :

$$
T=\operatorname{Th}(\mathfrak{M}, m)_{m \in M}+\left\{\text { "c } \cap \alpha=a_{\alpha} ": \alpha \in \operatorname{Ord}(\mathfrak{M})\right\} .
$$

We wish to show that $T$ has a model in which each of the 1-types in the following countable families is omitted:

- $\Gamma_{m}(x)=\{x \in m\} \cup\{x \neq a: a E m\}$, where $m \in M$;

- $\Lambda(x)=\{$ “ $x \in$ Ord" $\} \cup\left\{x \neq \tau(c): \tau(v)\right.$ is a term of $\left.(\mathfrak{M}, m)_{m \in M}\right\}$;

- for each term $\tau(x)$ of $(\mathfrak{M}, m)_{m \in M}$,

$$
\begin{aligned}
\Theta_{\tau}(x)=\{ & \{(c)=x \wedge \text { “ } x \in \operatorname{Ord} "\} \cup\left\{\tau(c) \notin V_{\alpha}: \alpha \in \operatorname{Ord}(\mathfrak{M})\right\} \\
& \left.\cup\left\{c \notin V_{\sigma(\tau(c)}\right): \sigma \text { is a term of }(\mathfrak{M}, m)_{m \in M}\right\}
\end{aligned}
$$


Note that if $\mathfrak{N}$ is any model of $T$ omitting each $\Gamma_{m}(x)$ then $\mathfrak{M} \prec \mathrm{e}$, and furthermore, that if $\mathfrak{N}$ omits $\Lambda(x)$ then every ordinal of $\mathfrak{N}$ is definable in $(\mathfrak{N}, c, m)_{m \in M}$. If, in addition, $\mathfrak{N}$ also omits all types of the form $\Theta_{\tau}(x)$ then $\mathfrak{N}$ will be an e-minimal e.e.e. of $\mathfrak{M}$. By the omitting types theorem, it suffices to show that each of the above types is locally omitted by $T$, i.e., for any formula $\psi(x)$ in the language of $T$, if $T+\exists x(\psi(x))$ is consistent then $T+\psi(x)$ fails to prove all of the formulas of the type in question. In order to do so, we first need a useful little lemma:

Lemma a. A sentence $\psi(c)$ is consistent with $T$ iff $(\mathfrak{M}, X) \vDash \forall \alpha \in$ Ord $\exists y(y \cap \alpha=X \cap \alpha \wedge \psi(y))$.

Proof. Suppose $\psi(c)$ is consistent with $T$, and that on the contrary, for some ordinal $\alpha_{0}$ of $\mathfrak{M}$,

$$
(\mathfrak{M}, X) \vDash \forall y\left(y \cap \alpha_{0}=X \cap \alpha_{0} \rightarrow \neg \psi(y)\right) .
$$

This implies that the sentence " $\forall y\left(y \cap \alpha_{0}=a_{\alpha_{0}} \rightarrow \neg \psi(y)\right)$ " is in $T$, which along with the fact that both of the sentences " $c \cap \alpha_{0}=a_{\alpha_{0}}$ " and $\psi(c)$ are members of $T$, contradicts our assumption of consistency of $T \cup\{\psi(c)\}$.

Now suppose $\psi(c)$ is inconsistent with $T$, i.e., that for some finite subset $T_{0}$ of $T, T_{0} \vdash \neg \psi(c)$. This immediately implies that for some ordinal $\alpha_{0}$ of $\mathfrak{M}, \operatorname{Th}(\mathfrak{M}, m)+" c \cap \alpha_{0}=a_{\alpha_{0}}$ " $+\psi(c)$ is inconsistent, which in turn implies that

$$
\mathfrak{M} \vDash \forall y\left(y \cap \alpha_{0}=a_{\alpha_{0}} \rightarrow \neg \psi(y)\right) .
$$

Therefore $(\mathfrak{M}, X)$ fails to satisfy $\forall \alpha \in \operatorname{Ord} \exists y(y \cap \alpha=X \cap \alpha$ and $\psi(y))$.

Motivated by Lemma $\boldsymbol{\beta}$, we introduce the following definable quantifier $Q$ in $(M, X)$ in order to considerably simplify the arguments to follow:

- $(\mathfrak{M}, X) \vDash Q y(\psi(y))$ iff $(\mathfrak{M}, X) \vDash \forall \alpha \in \operatorname{Ord} \exists y(y \cap \alpha=X \cap \alpha \wedge \psi(y))$.

Hence Lemma \& says that $\psi(c)$ is consistent with $T$ iff $(\mathfrak{M}, X) \vDash$ $Q y(\psi(y))$. To show that $\Gamma_{m}(x)$ is locally omitted by $T$, suppose $\exists x(\psi(x, c))$ is consistent with $T$, and that $T+\psi(x, c) \vdash x \in m$. Applying Lemma os we obtain

$$
(\mathfrak{M}, X) \vDash Q y \exists x \in m(\psi(x, y)) .
$$

Invoking the replacement scheme in $\mathrm{ZF}(X)$, there exists some $m_{0}$ in $M$ such that

$$
(\mathfrak{M}, X) \vDash Q y\left(\psi\left(m_{0}, y\right)\right),
$$

which implies that $T+\psi\left(m_{0}, c\right)$ is consistent. Therefore $T$ locally omits $\Gamma_{m}(x)$.

Now we look at the type $\Lambda(x)$. Again suppose $\exists x(\psi(x, c))$ is consistent with $T$, and that $T+\psi(x, c) \vdash$ " $x \in$ Ord". Therefore, Lemma os yields

$$
(\mathfrak{M}, X) \vDash Q y \exists x \in \operatorname{Ord}(\psi(x, y)) .
$$


Let $\tau_{0}(y)$ denote the definable term "the least ordinal $x$ such that $\psi(x, y)$ if it exists, and 0 otherwise"; then

$$
(\mathfrak{M}, X) \vDash Q y\left(\psi\left(\tau_{0}(y), y\right)\right),
$$

which implies, by Lemma $\boldsymbol{\beta}$, that $T$ is consistent with $\psi\left(\tau_{0}(c), c\right)$. So $T$ locally omits $\Lambda(x)$.

Finally we look at the type $\Theta_{\tau}$. The argument in this case is considerably more involved than the previous two cases. For the last time, suppose $\exists x(\psi(x, c))$ is consistent with $T$. Without loss of generality we may assume that for each ordinal $\alpha$ of $\mathfrak{M}$,

$$
T+\psi(x, c) \vdash\left(\tau(c)=x \wedge \text { " } x \in \text { Ord" } \wedge x \notin V_{\alpha}\right) .
$$

Let $\varphi(y)$ denote $\exists x(\psi(x, y) \wedge \tau(y)=x \wedge$ " $x \in$ Ord" $)$. Lemma implies

$$
(\mathfrak{M}, X) \vDash \forall \alpha \in \text { Ord } Q y\left(\varphi(y) \wedge \tau(y) \notin V_{\alpha}\right) .
$$

We will now use simultaneous transfinite recursion within $(\mathfrak{M}, X)$ to define three increasing sequences of ordinals of $\mathfrak{M},\left(r_{\gamma}: \gamma \in\right.$ Ord $),\left(s_{\gamma}: \gamma \in\right.$ Ord), and $\left(\alpha_{\gamma}: \gamma \in\right.$ Ord), as follows: to begin with, set $r_{0}=s_{0}=\alpha_{0}=0$. Suppose $\left(r_{\gamma}: \gamma<\beta\right)$ and $\left(s_{\gamma}: \gamma<\beta\right)$ and $\left(\alpha_{\gamma}: \gamma<\beta\right)$ have been defined for some ordinal $\beta$. Thanks to the replacement scheme, $\tau\left(\bigcup_{\gamma<\beta} V_{r_{\gamma}}\right)$ is bounded in Ord by some ordinal $\alpha_{\beta}$. Therefore, by (1) there is some $y$ such that $y \cap \beta=X \cap \beta, \varphi(y)$ holds, and $\tau(y) \notin V_{\alpha_{\beta}}$. Let $r_{\beta}$ be the rank of a minimal such $y$, and let $s_{\beta}$ denote the least ordinal of the form $\tau(y)$ for such minimal $y$ 's. More formally:

- $\alpha_{\beta}=\min \left\{\alpha \in\right.$ Ord : $\left.\alpha \notin \tau\left(\bigcup_{\gamma<\beta} V_{r_{\gamma}}\right)\right\}$,

- $r_{\beta}=\min \left\{\varrho(y): y \cap \beta=X \cap \beta \wedge \varphi(y) \wedge \tau(y) \notin V_{\alpha_{\beta}}\right\}$, and

- $s_{\beta}=\min \left\{\tau(y): \varrho(y)=r_{\beta} \wedge(y \cap \beta=X \cap \beta) \wedge \varphi(y) \wedge \tau(y) \notin V_{\alpha_{\beta}}\right\}$.

Therefore, if $R(y)$ denotes the formula

$$
\exists \beta \in \operatorname{Ord}\left(y \cap \beta=X \cap \beta \wedge \psi(\tau(y), y) \wedge \tau(y)=s_{\beta} \wedge y \in V_{r_{\beta}}\right) .
$$

then

$$
(\mathfrak{M}, X) \vDash Q y(R(y)) .
$$

Furthermore, if $\sigma(x)$ is the term defined by $\sigma(x)= \begin{cases}\min \left\{\varrho(y): y \cap \beta=X \cap \beta \wedge \psi(\tau(y), y) \wedge \tau(y)=s_{\beta}\right\} & \text { if } \exists \beta\left(x=s_{\beta}\right), \\ 0 & \text { otherwise }\end{cases}$ then

$$
(\mathfrak{M}, X) \vDash \forall y\left(R(y) \rightarrow\left(\psi(\tau(y), y) \wedge y \in V_{\sigma(\tau(y))}\right)\right) .
$$

Therefore, by (2),

$$
(\mathfrak{M}, X) \vDash Q y\left(\psi(\tau(y), y) \wedge y \in V_{\sigma(\tau(y))}\right) .
$$


Thanks to Lemma o, we can conclude that $T+\psi(x, c)+\left(c \in V_{\sigma(\tau(y))}\right)$ is consistent, establishing that $T$ locally omits $\Theta_{\tau}$.

We are finally ready to end the proof of Theorem 4.1. For any countable model $\mathfrak{M}$ of ZF, by Lemma 4.1.3 there are e-minimal e.e.e.'s $\mathfrak{N}$ of $\mathfrak{M}$ from which $\mathfrak{M}$ itself can be unambiguously recovered. Therefore for any such e.e.e. $\mathfrak{N}=(N, F)$ we can unambiguously refer to the countable family of first order theories $\mathcal{F}(\mathfrak{N})$, where

$$
\mathcal{F}(\mathfrak{N})=\left\{\operatorname{Th}(\mathfrak{M}, X): X \subseteq M \text { and } X=a_{F} \cap M \text { for some } a \in N\right\} .
$$

Note that $\mathcal{F}(\mathfrak{N})$ only depends on the isomorphism type of $\mathfrak{N}$. Lemmas 4.1 .2 and 4.1.3 together imply

$$
\mid \bigcup\{\mathcal{F}(\mathfrak{N}): \mathfrak{N} \text { is an e-minimal e.e.e. of } \mathfrak{M}\} \mid=2^{\aleph_{0}},
$$

which immediately establishes the existence of a continuum of pairwise nonisomorphic countable e.e.e.'s of extensions of $\mathfrak{M}$.

We can now calculate the number of nonisomorphic countable models whose ordinal standard parts are equal to a prescribed ordinal $\alpha$ (see the fourth clause of Definition 2.1).

Corollary 4.2. If a complete extension $T$ of $Z F$ has a model $\mathfrak{M}$ with $\operatorname{osp}(\mathfrak{M})=\alpha<\omega_{1}$, then $T$ has $2^{\aleph_{0}}$ nonisomorphic countable models whose ordinal standard parts are equal to $\alpha$.

Proof. Suppose $\mathfrak{M}$ is a model of $T$ with $\operatorname{osp}(\mathfrak{M})=\alpha<\omega_{1}$. If $\mathfrak{M}$ is not well-founded then any e.e.e. of $\mathfrak{M}$ will have the same standard part as $\mathfrak{M}$, in which case we are done by Theorem 4.1. If $\mathfrak{M}$ is well-founded, it suffices to first build an elementary end extension $\mathfrak{N}$ of $\mathfrak{M}$ for which $\operatorname{Ord}(\mathfrak{N}) \backslash \operatorname{Ord}(\mathfrak{M})$ has no least element (which is possible by a result of Hutchinson $[\mathrm{Hu}]$ ) and then invoke Theorem 4.1 to obtain a continuum of e.e.e.'s of $\mathfrak{N}$ all of which have $\alpha$ as their ordinal standard part.

Corollary 4.3 below generalizes a result of Vetulani [V] who constructed two nonisomorphic elementary equivalent nonstandard models of $\mathrm{ZF}+\mathbf{V}$ $=\mathbf{L}$ whose sets of ordinals are isomorphic. Vetulani's construction uses the infinitary techniques of Friedman [Fr-1] and only works for order types $\tau$ such that $\operatorname{osp}(\tau)=\alpha>\omega$ for some recursively inaccessible ordinal $\alpha$.

COROllary 4.3. If $\tau$ is a countable linear order which is not wellordered and $T$ is a complete extension of $Z F$, then $\mu(T, \tau) \in\left\{0,2^{\aleph_{0}}\right\}$.

Proof. Suppose $\tau$ is a countable linear order which is not well-ordered and let $\mathfrak{M} \vDash \mathrm{ZF}$ with $\tau \cong \operatorname{Ord}(\mathfrak{M})$. By Friedman's characterization of order types of countable nonstandard models [Fr-1, Sec. 3], if $\mathfrak{M}$ and $\mathfrak{N}$ are countable nonstandard models of the Kripke-Platek set theory KP with the same ordinal standard part $\alpha$, then 
- if $\alpha>\omega$ then $\operatorname{Ord}(\mathfrak{M}) \cong \operatorname{Ord}(\mathfrak{N}) \cong \alpha(1+\eta)$, where $\eta$ is the order type of rationals, and

- if $\alpha=\omega$ and $\operatorname{SSy}(\mathfrak{M})=\operatorname{SSy}(\mathfrak{N})$ then $\operatorname{Ord}(\mathfrak{M}) \cong \operatorname{Ord}(\mathfrak{N})$.

In light of Friedman's characterization and the fact that $\operatorname{osp}(\mathfrak{M})$ and $\operatorname{SSy}(\mathfrak{M})$ are both invariant under e.e.e.'s when $\mathfrak{M}$ is nonstandard, $\operatorname{Ord}(\mathfrak{M})$ $\cong \operatorname{Ord}(\mathfrak{N})$ for each countable e.e.e. $\mathfrak{N}$ of $\mathfrak{M}$. Therefore by Theorem 4.1 the proof is complete.

\section{Open questions and further results}

Question 5.1. Can $1<\mu(T, \alpha)<2^{\aleph_{0}}$ hold for some completion $T$ of $Z F$ and some countable ordinal $\alpha$ ?

- By Theorem 3.5 such a $T$ has to prove $\mathbf{V}=\mathbf{O D}$, and by Theorem 3.6, $L_{\alpha}$ cannot be pointwise definable. Moreover, by Remark 3.6.1 if $\alpha$ is the height of the pointwise definable model of $T$, then $L_{\alpha}$ must contain completely ineffable cardinals which are totally indescribable.

QUESTION 5.2. What is the behavior of $\mu(T, \alpha)$ as a function of $\alpha$ ? In particular, is it possible to have $\alpha<\beta$ but $\mu(T, \alpha)>\mu(T, \beta)>0$ ?

- Recall from Remark 3.10(b) that it is possible to have $\alpha_{T}<\alpha$ with $\mu\left(T, \alpha_{T}\right)=1$ and $\mu(T, \alpha)=2^{\aleph_{0}}$.

Question 5.3. If $T \vdash \mathbf{V}=\mathbf{O D}$ and $T$ has a well-founded model with $\mu\left(T, \alpha_{T}\right)>1$ (where $\alpha_{T}$ is the height of the pointwise model of $T$ ), then does $T$ prove " 0 " exists"?

- This is motivated by parts (a) and (b) of Theorem 3.8, and Remark 3.9.

Question 5.4. Do Corollaries 4.2 and 4.3 remain valid if $Z F$ is replaced with the Kripke-Platek set theory KP, or with the Kelley-Morse theory of classes KM?

- We can show that Theorem 4.1, and therefore Corollaries 4.2 and 4.3, remain valid for complete extensions of "ZF - Powerset + Global Choice" using a different argument. We suspect that a positive answer to Question 5.4 for KP can be obtained by working with $\Sigma_{1}$-e.e.e.'s, at least for theories which have $\Sigma_{1}$-definable Skolem functions for $\Sigma_{1}$-formulas (such theories include any completion of $\mathrm{KP}+\mathbf{V}=\mathbf{L}$, by Jensen's work).

QUESTION 5.5. If $\alpha$ is an uncountable ordinal and $T$ is a completion of $Z F$, what can be said about the number of transitive models of $T$ of cardinality $|\alpha|$ ?

QUESTION 5.6. Are there uncountable analogues of Corollaries 4.2 and 4.3 ? 
- The analogue of Proposition 1.1(b) is true for all uncountable cardinals $\kappa$ as a corollary of Shelah's theorem [Sh] on the existence of $2^{\kappa}$ nonisomorphic models of power $\kappa$ for any unstable theory.

- Shelah's argument can be combined with Keisler and Morley's techniques $[\mathrm{KM}]$ to produce $2^{\kappa}$ nonisomorphic models of power $\kappa$ all of which are e.e.e.'s of a prescribed countable model of $Z F$. As a result, if $T$ is a completion of ZF which has a model $\mathfrak{M}$ with $\operatorname{osp}(\mathfrak{M})=\alpha<\omega_{1}$ then for each uncountable cardinal $\kappa$ there are $2^{\kappa}$ nonisomorphic models of power $\kappa$ of $T$ all of whose ordinal standard parts are $\alpha$.

- Using iterated ultrapowers along carefully chosen non-well-founded linear orders, we can show that if $T$ is an extension of ZFC+ "there is a proper class of measurable cardinals" which has a model $\mathfrak{M}$ with $\operatorname{osp}(\mathfrak{M})=\alpha \leq \kappa$, then $T$ has $2^{\kappa}$ nonisomorphic models of power $\kappa$ all of whose ordinal standard parts are $\alpha$, where $\kappa$ is any prescribed uncountable cardinal.

- A general result of Keisler [Ke, Section 31] implies that every completion of ZF has $2^{\aleph_{1}}$ nonisomorphic $\aleph_{1}$-like models. However, as shown in [E-3] assuming $\mathrm{Con}(\mathrm{ZF}+$ there exists an $\omega$-Mahlo cardinal) there are models of set theory in which the only $\aleph_{2}$-like models of ZFC are those satisfying the recursive set of axioms $\Phi$, where

$\Phi=\left\{" \exists \kappa\left(\kappa\right.\right.$ is $n$-Mahlo and $V_{\kappa}$ is a $\Sigma_{n}$-elementary submodel of $\left.\mathbf{V}\right) "$ :

$$
n \in \omega\} \text {. }
$$

Furthermore, one can show that each completion of ZFC $+\Phi$ has $2^{\kappa}$ nonisomorphic $\kappa$-like models, where $\kappa$ is any prescribed uncountable cardinal.

\section{References}

[Ba] J. Barwise, Admissible Sets and Structures, Springer, Berlin, 1975.

[Be] J. Bell, Boolean-Valued Models and Independence Proofs in Set Theory, Oxford Univ. Press, Oxford, 1985.

[CK] C. C. Chang and H. J. Keisler, Model Theory, North-Holland, Amsterdam, 1973.

[C] P. Cohen, Set Theory and the Continuum Hypothesis, Benjamin, New York, 1966.

[E-1] A. Enayat, Weakly compact cardinals in models of set theory, J. Symbolic Logic 50 (1985), 476-486.

[E-2] -, Definable elements and undefinable classes of models of set theory and arithmetic, Proc. Amer. Math. Soc. 103 (1988), 1216-1220.

[E-3] - Power-like models of set theory, J. Symbolic Logic 66 (2001), 1766-1782.

[Fe] U. Felgner, Comparisons of the axioms of local and universal choice, Fund. Math. 71 (1971), 43-62.

[Fr-1] H. Friedman, Countable models of set theories, in: Cambridge Summer School in Mathematical Logic (Cambridge, 1971), H. Rogers and A. R. D. Mathias (eds.), Lecture Notes in Math. 337, Springer, Berlin, 1973, 539-573. 
[Fr-2] H. Friedman, Large models of countable height, Trans. Amer. Math. Soc. 201 (1975), 227-239.

[He] J. van Heijenoort, From Frege to Gödel, Harvard Univ. Press, Cambridge, 1967.

$[\mathrm{Hu}] \mathrm{J}$. Hutchinson, Elementary extensions of countable models of set theory, J. Symbolic Logic 41 (1976), 139-145.

[J] T. Jech, Set Theory, Academic Press, New York, 1978.

[JMMP] T. Jech, M. Magidor, W. Mitchell and K. Prikry, Precipitous ideals, J. Symbolic Logic 45 (1980), 1-8.

[JS] R. Jensen and R. Solovay, Applications of almost disjoint forcing, in: Mathematical Logic and Foundations of Set Theory, Y. Bar-Hillel (ed.), NorthHolland, Amsterdam, 1970, 84-104.

[Ka] A. Kanamori, The Higher Infinite, Springer, Berlin, 1994.

[Ke] H. J. Keisler, Model Theory for Infinitary Logic, North-Holland, Amsterdam, 1971.

$[\mathrm{KM}] \quad$ H. J. Keisler and M. Morley, Elementary extensions of models of set theory, Israel J. Math. 6 (1968), 49-65.

[Kl] E. M. Kleinberg, A combinatorial characterization of normal M-ultrafilters, Adv. Math. 30 (1978), 77-84.

[KP] R. Kossak and J. Paris, Subsets of models of arithmetic, Arch. Math. Logic 32 (1992), 65-73.

[Ku] K. Kunen, Set Theory, North-Holland, Amsterdam, 1980.

[MD] R. Mansfield and J. Dawson, Boolean-valued set theory and forcing, Synthèse 33 (1976), 223-252.

[Mc] K. McAloon, Consistency results about ordinal definability, Ann. Math. Logic 2 (1971), 449-467.

[Mi] A. Miller, Descriptive Set Theory and Forcing, Lecture Notes in Logic 4, Springer, Amsterdam, 1995.

[Mor] M. Morley, The number of countable models, J. Symbolic Logic 35 (1970), $14-18$.

[Mos] A. Mostowski, A remark on models of the Gödel-Bernays axioms for set theory, in: Sets and Classes, G. Müller (ed.), North-Holland, Amsterdam, 1976, 325-340.

[Re] J. P. Ressayre, Modèles non standard et un sous-système remarquable de $Z F$, in: Modèles non standard en arithmétique et théorie des ensembles, J. P. Ressayre and A. Wilke (eds.), Publ. Math. Univ. Paris 7, Paris, 1986.

[Ro] S. Roguski, Extensions of models of ZFC to models of $Z F+V=H O D$ with applications, in: Set Theory and Hierarchy Theory, W. Marek et al. (eds.), Lecture Notes in Math. 537, Springer, Berlin, 1976, 241-247.

[Sc] J. Schlipf, Recursively saturated models of set theory, Proc. Amer. Math. Soc. 80 (1980), 135-142.

[Sh] S. Shelah, On the number of models of an unstable first order theory, Israel J. Math. 9 (1971), 473-487.

[Si] S. Simpson, Forcing and models of arithmetic, Proc. Amer. Math. Soc. 43 (1974), 93-194.

[Sk] T. Skolem, Einige Bemerkungen zur axiomatischen Begrundung der Mengenlehre, in: Proc. 5th Scandinavian Congress (Helsinki, 1922), Akademiska Bokhanden, Helsinki, 1923, 217-232; English transl. in [He].

[Sm] C. Smoryński, Lectures on nonstandard models of arithmetic, in: Logic Colloquium '82, G. Lolli et al. (eds.), North-Holland, Amsterdam, 1984, 1-70. 
[So] R. Solovay, A model of set theory in which every set of reals is Lebesgue measurable, Ann. of Math. 92 (1970), 1-56.

[V] Z. Vetulani, Categoricity relative to ordinals for models of set theory and the nonabsoluteness of $L$, in: Set Theory and Hierarchy Theory, W. Marek et al. (eds.), Lecture Notes in Math. 537, Springer, Berlin, 1976, 285-290.

[W-1] H. Woodin, $\boldsymbol{\Sigma}_{1}^{2}$-absoluteness and supercompact cardinals, circulated hand-written notes, 1985.

[W-2] - The Axiom of Determinacy, Forcing Axioms, and the Nonstationary Ideal, de Gruyter, Berlin, 1999.

Department of Mathematics and Statistics

American University

Washington, DC 20016-8050, U.S.A.

E-mail: enayat@american.edu

Received 22 March 2001;

in revised form 5 February 2002 